\title{
Primitivity and Independent Sets in Direct Products of Vertex-Transitive Graphs *
}

\author{
Huajun Zhang \\ Department of Mathematics, Shanghai Normal University, Shanghai 200234, China \\ and \\ Department of Mathematics, Zhejiang Normal University, Jinhua 321004, P.R. China \\ E-mail: huajunzhang@zjnu.cn
}

\begin{abstract}
We introduce the concept of the primitivity of independent set in vertex-transitive graphs, and investigate the relationship between the primitivity and the structure of maximum independent sets in direct products of vertex-transitive graphs. As a consequence of our main results, we positively solve an open problem related to the structure of independent sets in powers of vertex-transitive graphs.
\end{abstract}

\section{Introduction}

The direct product $G \times H$ of two graphs $G$ and $H$ is defined by

$$
V(G \times H)=V(G) \times V(H)
$$

and

$$
E(G \times H)=\left\{\left[\left(u_{1}, u_{2}\right),\left(v_{1}, v_{2}\right)\right]:\left[u_{1}, v_{1}\right] \in E(G) \text { and }\left[u_{2}, v_{2}\right] \in E(H)\right\} .
$$

For a graph $G$, let $G^{n}=G \times \cdots \times G$ denote the $n$-th power of $G$.

It is clear that if $I$ is an independent set of $G$ (or $H)$, then $I \times H($ or $G \times I)$ is an independent set of $G \times H$. We say that $G \times H$ is MIS-normal (maximumindependent-set-normal) if each of its maximum independent sets is of this form.

* Supported by the National Natural Science Foundation of China (No.10826084) and Zhejiang Innovation Project (Grant No. T200905). 
Then the independence number

$$
\alpha(G \times H)=\max \{\alpha(G)|H|, \alpha(H)|G|\}
$$

if $G \times H$ is MIS-normal. A product $G_{1} \times G_{2} \times \cdots \times G_{n}$ is said to be MIS-normal if all of its maximum independent sets are preimages of projections of maximum independent sets of one of its factors.

This poses two immediate problems: whether (1) holds for all graphs $G$ and $H$, and whether $G \times H$ is MIS-normal when (1) holds. In general, however, (1) does not hold for some non-vertex-transitive graphs (see [7]). So, Tardif [3] asked whether (1) holds for all vertex-transitive graphs $G$ and $H$. Larose and Tardif [2] investigated the relationship between the projectivity and the structure of maximal independent sets in powers of a circular graph, Kneser graph, or truncated simplex. Recently, Mario and Vera [5] proved that (1) holds for some special vertex-transitive graphs, e.g., circular graphs and Kneser graphs. In fact, Frankl [6] proved in 1996, one year before Tardif's question was posed, that (1) holds for Kneser graphs. Subsequently, Ahlswede, Aydinian and Khachatrian [8] generalized Frankl's result.

In the context of vertex-transitive graphs, the "No-Homomorphism" lemma of Albertson and Collins [1] is useful to get bounds on the size of independent sets.

Lemma 1.1 ([1]) Let $G$ and $H$ be two graphs such that $G$ is vertex-transitive and there exists a homomorphism $\phi: H \mapsto G$. Then $\frac{\alpha(G)}{|V(G)|} \leq \frac{\alpha(H)}{|V(H)|}$, and the equality holds if and only if for any independent set I of cardinality $\alpha(G)$ in $G, \phi^{-1}(I)$ is an independent set of cardinality $\alpha(H)$ in $H$.

By this lemma, it is easy to deduce that $\alpha\left(G^{n}\right)=\alpha(G)|V(G)|^{n-1}$ for any vertextransitive graph $G$ and positive integer $n$ (see [2]). So it is natural to ask whether $G^{n}$ is MIS-normal. Evidently, if $G^{n}$ is MIS-normal for some $n>2$, so is $G^{2}$. Conversely, Larose and Tardif [2] posed the following problem.

Problem 1.2 (see [2] Let $G$ be a non-bipartite vertex-transitive graph. If $G^{2}$ is $M I S$-normal, is the same for all powers of $G$ ?

This paper is organized as follows. In the next section, we introduce a concept of the primitivity of independent sets in a vertex-transitive graph, and prove that the 
primitivity can be preserved in direct products under certain conditions. Based on these results we establish in section 3 a direct product theorem on the MIS-normality. As a consequence, Problem 1.2 is solved.

\section{Primitivity of independent sets}

In the sequel of this paper, let $G$ and $H$ be vertex-transitive graphs. By $I(G)$ we denote the set of all maximum independent sets of $G$. For any subset $A$ of $V(G)$, let $\alpha(A)$ denote the independence number of the induced subgraph of $G$ by $A$, and we define

$$
\begin{gathered}
N_{G}(A)=\{b \in G:(a, b) \in E(G) \text { for some } a \in A\}, \\
N_{G}[A]=N_{G}(A) \cup A \text { and } \bar{N}_{G}[A]=G-N_{G}[A] .
\end{gathered}
$$

In Lemma 1.1, by taking $H$ as an induced subgraph of $G$ and $\phi$ as the embedding mapping, we obtain the following lemma (cf. 4]).

Lemma 2.1 $\frac{\alpha(G)}{|V(G)|} \leq \frac{\alpha(B)}{|B|}$ holds for all $B \subseteq V(G)$. Equality implies that $|S \cap B|=$ $\alpha(B)$ for every $S \in I(G)$.

A graph $G$ is said to be non-empty if $E(G) \neq \emptyset$. Lemma 2.1 implies that $\alpha(G) \leq|V(G)| / 2$ for all non-empty vertex-transitive graphs. Equality holds if and only if $G$ is bipartite, which we state as a corollary for reference.

Corollary 2.2 Let $G$ be a non-empty vertex-transitive graph. Then $\frac{\alpha(G)}{|G|} \leq \frac{1}{2}$, and equality holds if and only if $G$ is bipartite.

Proposition 2.3 Let $A$ be an independent set of $G$. Then $\frac{|A|}{\left|N_{G}\right| A||} \leq \frac{\alpha(G)}{|V(G)|}$. Equality implies that $\left|S \cap N_{G}[A]\right|=|A|$ for every $S \in I(G)$, and in particularly $A \subseteq S$ for some $S \in I(G)$.

Proof. Since $A$ is an independent set, clearly

$$
\frac{|A|+\alpha\left(\bar{N}_{G}[A]\right)}{\left|N_{G}[A]\right|+\left|\bar{N}_{G}[A]\right|} \leq \frac{\alpha(G)}{|V(G)|}
$$


By Lemma 2.1 we see that $\frac{\alpha\left(\bar{N}_{G}[A]\right)}{\left|\bar{N}_{G}[A]\right|} \geq \frac{\alpha(G)}{|V(G)|}$, so $\frac{|A|}{\left|N_{G}[A]\right|} \leq \frac{\alpha(G)}{|V(G)|}$. Equality in the latter implies equality in the former. In this case any $S \in I(G)$ must be the union of a maximum independent set in $\bar{N}_{G}[A]$ and an independent set of size $|A|$ in $N_{G}[A]$, and thus $\left|S \cap N_{G}[A]\right|=|A|$.

An independent set $A$ in $G$ is said to be imprimitive if $|A|<\alpha(G)$ and $\frac{|A|}{\left|N_{G}[A]\right|}=$ $\frac{\alpha(G)}{|V(G)|}$. We say that $G$ is $I S$-imprimitive if $G$ has an imprimitive independent set. In the other case, $G$ is $I S$-primitive.

Proposition 2.4 Let $A$ be a maximum imprimitive independent set of $G$. Set $B=$ $\bar{N}_{G}[A]$. Then $\frac{\alpha(B)}{|B|}=\frac{\alpha(G)}{|V(G)|}$ and $\{\sigma(B) \mid \sigma \in A u t(G)\}$ forms a nontrivial partition of $V(G)$, i.e., $\sigma(B) \cap B=\emptyset$ or $B$ for each $\sigma \in A u t(G)$.

Proof. Clearly $\frac{|A|+\alpha(B)}{\left|N_{G}[A]\right|+|B|} \leq \frac{\alpha(G)}{|V(G)|}$. Combining the condition of $A$ and Lemma 2.1, we have $\frac{\alpha(B)}{|B|}=\frac{\alpha(G)}{|V(G)|}$. By definition, $N_{G}[\sigma(A)]=\sigma\left(N_{G}[A]\right)$ for all $\sigma \in \operatorname{Aut}(G)$. Suppose that there exists a $\sigma \in \operatorname{Aut}(G)$ such that $\sigma(B) \neq B$ and $\sigma(B) \cap B \neq \emptyset$. Then $\sigma\left(N_{G}[A]\right) \neq N_{G}[A]$ and

$$
|V(G)|>\left|N_{G}[A] \cup \sigma\left(N_{G}[A]\right)\right|>\left|N_{G}[A]\right|
$$

Let $C=\sigma(A) \cup\left(A-N_{G}[\sigma(A)]\right)$. Then $C$ is also an independent set and

$$
N_{G}[C] \subseteq N_{G}[A] \cup \sigma\left(N_{G}[A]\right)
$$

By Proposition 2.3, $\left|S \cap N_{G}[A]\right|=|A|$ for all $S \in I(G)$, which implies that $(S-$ $\left.N_{G}[A]\right) \cup A \in I(G)$ for all $S \in I(G)$. Similarly,

$$
\begin{aligned}
& \left.\left(\left(S-N_{G}[A]\right) \cup A\right)-N_{G}[\sigma(A)]\right) \cup \sigma(A) \\
= & \left(S-N_{G}[A] \cup N_{G}[\sigma(A)]\right) \cup\left(A-N_{G}[\sigma(A)]\right) \cup \sigma(A) \\
= & \left(S-N_{G}[A] \cup N_{G}[\sigma(A)]\right) \cup C
\end{aligned}
$$

is also a maximum independent set of $G$, which implies $\left|S \cap\left(N_{G}[A] \cup N_{G}[\sigma(A)]\right)\right|=$ $|C|$ for all $S \in I(G)$.

Given a $u \in V(G)$, suppose that there are $r$ 's in $I(G)$ such that $u \in S$. Since $G$ is vertex-transitive, the number $r$ is independent of the choice of $u$. Thus $r|V(G)|=\alpha(G)|I(G)|$. On the other hand, since $\left|S \cap\left(N_{G}[A] \cup N_{G}[\sigma(A)]\right)\right|=|C|$ for 
all $S \in I(G),|C||I(G)|=r\left|N_{G}[A] \cup N_{G}[\sigma(A)]\right|$. Combining the above two equalities, we have $\frac{|C|}{\left|N_{G}[A] \cup N_{G}[\sigma(A)]\right|}=\frac{\alpha(G)}{|V(G)|}$. Thus, by Proposition 2.3 we have

$$
\frac{\alpha(G)}{|V(G)|} \geq \frac{|C|}{N_{G}[C]} \geq \frac{|C|}{\left|N_{G}[A] \cup N_{G}[\sigma(A)]\right|}=\frac{\alpha(G)}{|V(G)|},
$$

which implies $N_{G}[C]=N_{G}[A] \cup N_{G}[\sigma(A)]$ and $\frac{|C|}{\left|N_{G}[C]\right|}=\frac{\alpha(G)}{|V(G)|}$. By (2), we have $|A|<|C|<\alpha(G)$, contradicting the maximality of $|A|$. This completes the proof.

The concept of primitivity comes from permutation groups: A permutation group $\Gamma$ acting on a set $X$ is called primitive if $\Gamma$ preserves no nontrivial partition of $X$. In the other case, $\Gamma$ is imprimitive. As usual (see e.g. [2]), a vertex-transitive graph $G$ is called primitive if its automorphism group, as a permutation group on $V(G)$, is primitive. By Proposition 2.4 we see that if $G$ is primitive, then $G$ is IS-primitive. But the converse is not true.

For any $S \subseteq V(G) \times V(H), a \in G$ and $u \in H$, define

$$
\partial_{G}(u, S)=\{b \in G:(b, u) \in S\}, \quad \partial_{H}(a, S)=\{v \in H:(a, v) \in S\},
$$

and

$$
\partial_{G}(S)=\left\{b \in G: \partial_{H}(b, S) \neq \emptyset\right\}, \quad \partial_{H}(S)=\left\{v \in H: \partial_{G}(v, S) \neq \emptyset\right\} .
$$

By definition we see that $\partial_{G}(S)$ and $\partial_{H}(S)$ are in fact the projections of $S$ on $G$ and $H$, respectively.

Lemma 2.5 Suppose $G \times H$ is MIS-normal and $\frac{\alpha(H)}{|H|} \leq \frac{\alpha(G)}{|G|}$. If $G \times H$ is ISimprimitive, then one of the following two possible cases holds:

(i) $\frac{\alpha(H)}{|H|}=\frac{\alpha(G)}{|G|}$, and one of them is IS-imprimitive or both $G$ and $H$ are bipartite;

(ii) $\frac{\alpha(H)}{|H|}<\frac{\alpha(G)}{|G|}$, and $G$ is IS-imprimitive or $H$ is disconnected.

Proof. Throughout this proof, we denote $N_{G \times H}[A]$ by $N[A]$ for brevity. Suppose that $G \times H$ is IS-imprimitive and let $A$ be a maximum imprimitive independent set of $G \times H$. Clearly, $\alpha(G \times H)=\alpha(G)|V(H)|$, and thus $\frac{|A|}{|N[A]|}=\frac{\alpha(G \times H)}{|V(G \times H)|}=\frac{\alpha(G)}{|V(G)|}$. If $E(G)=\emptyset$, the result is trivial, so we suppose $E(G) \neq \emptyset$, then Corollary 2.2 implies that $\frac{\alpha(H)}{|V(H)|} \leq \frac{\alpha(G)}{|V(G)|} \leq \frac{1}{2}$. By Proposition 2.3, there exists some $S \in I(G \times H)$ such 
that $A=S \cap N[A]$. Since $G \times H$ is MIS-normal, we may assume that $S=S^{\prime} \times H$ for some $S^{\prime} \in I(G)$. Thus $A=\left(S^{\prime} \times H\right) \cap N[A]$. Set $B=\bar{N}[A]$. Then, by Proposition 2.4. $\sigma(B) \cap B=\emptyset$ or $B$ for every $\sigma \in \operatorname{Aut}(G \times H)$.

Set $C=\partial_{G}(B)$. For every pair $a$ and $b$ of $C$, select $u \in \partial_{H}(a, B)$ and $v \in$ $\partial_{H}(b, B)$. Since $G$ and $H$ are vertex-transitive, there exist $\gamma \in \operatorname{Aut}(G)$ and $\tau \in$ $\operatorname{Aut}(H)$ such that $a=\gamma(b)$ and $u=\tau(v)$. It is clear that $\sigma=(\gamma, \tau) \in \operatorname{Aut}(G \times H)$ and $(a, u)=\sigma(b, v) \in \sigma(B) \cap B$. By Proposition 2.4, we conclude that $\sigma(B)=B$. Thus, we have $\partial_{H}(a, B)=\tau\left(\partial_{H}(b, B)\right)$. Therefore, $\left.\left|\partial_{H}(a, B)\right|=\mid \partial_{H}(b, B)\right) \mid$ for any $a, b \in C$. In the following, we will complete the proof by two cases.

Case 1: $C \neq V(G)$. Set $\bar{C}=(V(G)-C)$. Then $(\bar{C} \times H) \cap B=\emptyset$, and thus $\bar{C} \times H \subseteq N[A]$. For every $S^{\prime \prime} \in I(G)$, it is clear that $S^{\prime \prime} \times H$ is a maximum independent set of $G \times H$. Since $\frac{\alpha(B)}{|B|}=\frac{\alpha(G \times H)}{|G \times H|}=\frac{\alpha(G)}{|V(G)|}$ and $\left|\partial_{H}(a, B)\right|=\left|\partial_{H}(b, B)\right|$ for all $a, b \in \partial_{G}(B)$, from Lemma 2.1 and the MIS-normality of $G \times H$ it follows that

$$
\frac{\left|\left(S^{\prime \prime} \times H\right) \cap B\right|}{|B|}=\frac{\left|S^{\prime \prime} \cap C\right|}{|C|}=\frac{\alpha(G)}{|V(G)|} .
$$

Thus for every $S^{\prime \prime} \in I(G)$,

$$
\frac{\alpha(G)}{|V(G)|}=\frac{\left|S^{\prime \prime}\right|}{|V(G)|}=\frac{\left|S^{\prime \prime} \cap C\right|+\left|S^{\prime \prime} \cap \bar{C}\right|}{|C|+|\bar{C}|}=\frac{\left|S^{\prime \prime} \cap \bar{C}\right|}{|\bar{C}|}=\frac{\left|S^{\prime \prime} \cap C\right|}{|C|} .
$$

Recall that $\bar{C} \times H \subseteq N[A]$ and $A \subseteq S^{\prime} \times H$, it is easy to see that $A=N[A] \cap\left(S^{\prime} \times H\right)$ and $\partial_{G}(A \cap(\bar{C} \times H))=S^{\prime} \cap \bar{C}$. Setting $F=S^{\prime} \cap \bar{C}$, we have that $a \times H \subseteq A$ for every $a \in F$. If $N_{G}[F] \cap C \neq \emptyset$, then there exist $a \in F$ and $b \in C$ such that $(a, b) \in E(G)$. Since $B=\bar{N}[A]$ and $a \times H \subseteq A$, by definition, $(b, u) \subseteq \bar{N}[a \times H]$ for every $u \in \partial_{H}(b, B)$. Hence $N_{H}[H] \neq \emptyset$ and $E(H)=\emptyset$, which contradicts that $\frac{\alpha(H)}{|H|} \leq \frac{1}{2}$. Thus $N_{G}[F] \cap C=\emptyset$, i.e., $N_{G}[F] \subseteq \bar{C}$. By Proposition 2.3 and (3)),

$$
\frac{\alpha(G)}{|V(G)|} \geq \frac{|F|}{\left|N_{G}[F]\right|}=\frac{\left|S^{\prime} \cap \bar{C}\right|}{\left|N_{G}[F]\right|} \geq \frac{\left|S^{\prime} \cap \bar{C}\right|}{|\bar{C}|}=\frac{\alpha(G)}{|V(G)|} .
$$

Therefore $\frac{|F|}{\left|N_{G}[F]\right|}=\frac{\alpha(G)}{|V(G)|}$, so $G$ is IS-imprimitive and (i) holds.

Case 2: $C=V(G)$. Since $\left.\left|\partial_{H}(a, B)\right|=\mid \partial_{H}(b, B)\right) \mid$ for all $a, b \in V(G)$, we have $\partial_{G}(N[A])=V(G)$ and $\left|\partial_{H}(a, N[A])\right|=\left|\partial_{H}(b, N[A])\right|<|H|$ for all $a, b \in V(G)$. Since $A=\left(S^{\prime} \times H\right) \cap N[A], \partial_{H}(a, N[A]) \subseteq \partial_{H}\left(a, S^{\prime} \times H\right)$ for all $a \in \partial_{G}(A)$. Thus $\partial_{H}(a, A)=\partial_{H}(a, N[A])$ for all $a \in \partial_{G}(A)$. Select two vertices $a$ and $b$ of $V(G)$ such that $a \in \partial_{G}(A)$ and $(a, b) \in E(G)$. Then, for every $u \in\left[V(H)-\partial_{H}(b, N[A])\right]$ and 
$v \in \partial_{H}(a, N[A])$, it is clear that $[(b, u),(a, v)] \notin E(G \times H)$, so $(u, v) \notin E(H)$. This means $u \notin N_{H}\left(\partial_{H}(a, N[A])\right)$, that is,

$$
V(H)-\partial_{H}(b, N[A]) \subseteq V(H)-N_{H}\left(\partial_{H}(a, N[A])\right)
$$

If $\partial_{H}(b, N[A])=\partial_{H}(a, N[A])$, it follows from (44) that $H$ is disconnected, and so either (i) or (ii) holds.

Suppose that $\partial_{H}(b, N[A]) \neq \partial_{H}(a, N[A])$ and set $D=\partial_{H}(a, N[A])-\partial_{H}(b, N[A])$. It is easy to check that

$$
2|D|=\left|\partial_{H}(a, N[A]) \cup \partial_{H}(b, N[A])-\partial_{H}(a, N[A]) \cap \partial_{H}(b, N[A])\right| .
$$

Since $D \subseteq H-\partial_{H}(b, N[A])$ and $D \subseteq \partial_{G}(a, N[A])$, by (44), we have

$$
D \subseteq V(H)-\partial_{H}(b, N[A]) \subseteq V(H)-N_{H}\left(\partial_{H}(a, N[A])\right) \subseteq V(H)-N_{H}(D)
$$

So $D$ is an independent set of $H$ and

$$
\begin{aligned}
N_{H}[D] & \subseteq D \cup\left[\partial_{H}(b, N[A])-\partial_{H}(a, N[A])\right] \\
& =\partial_{H}(a, N[A]) \cup \partial_{H}(b, N[A])-\partial_{H}(a, N[A]) \cap \partial_{H}(b, N[A]),
\end{aligned}
$$

which implies that $\frac{1}{2} \geq \frac{\alpha(H)}{|V(H)|} \geq \frac{|D|}{\left|N_{H}[D]\right|} \geq \frac{1}{2}$. Thus $\frac{\alpha(G)}{|V(G)|}=\frac{\alpha(H)}{|V(H)|}=\frac{1}{2}$. By Corollary 2.2, $G$ and $H$ are both bipartite, so (i) holds and the proof completed.

Theorem 2.6 Let $G$ and $H$ be two non-bipartite vertex-transitive graph such that $\frac{\alpha(H)}{|V(H)|}=\frac{\alpha(G)}{|V(G)|}$. If $G \times H$ is MIS-normal, then $G, H$ and $G \times H$ are all IS-primitive.

Proof. First, suppose that $G$ is IS-imprimitive and let $A$ be an imprimitive independent set in $G$. For any $S \in I(H)$, let $S^{\prime}=\left(\bar{N}_{G}[A] \times S\right) \cup(A \times H)$. It is clear that $S^{\prime}$ is an independent set of $G \times H$ and

$$
\begin{aligned}
\left|S^{\prime}\right| & =\left|\bar{N}_{G}[A] \alpha(H)\right|+|A||V(H)|=\left(\left|\bar{N}_{G}[A]\right|+\left|N_{G}[A]\right|\right) \alpha(H) \\
& =|V(G)| \alpha(H)=\alpha(G \times H),
\end{aligned}
$$

i.e., $S^{\prime}$ is a maximum independent set of $G \times H$, contradicting the MIS-normality of $G$. Therefore, $G$ is IS-primitive. Similarly, $H$ is also IS-primitive. By Lemma 2.5, $G \times H$ is IS-primitive. 


\section{MIS-normality of the Products of Graphs}

The following theorem is the main result on the MIS-normality of products of vertextransitive graphs in this paper.

Theorem 3.1 Let $G$ and $H$ be two vertex-transitive graphs. Suppose that there exists an induced subgraph $G^{\prime}$ of $G$ such that $G^{\prime} \times H$ is MIS-normal and $\frac{\alpha\left(G^{\prime}\right)}{\left|V\left(G^{\prime}\right)\right|}=$ $\frac{\alpha(G)}{|V(G)|}$. Then either: (i) $G \times H$ is MIS-normal, or (ii) $\frac{\alpha(G)}{|V(G)|}=\frac{\alpha(H)}{|V(H)|}$ and $G$ is IS-imprimitive, or (iii) $\frac{\alpha(G)}{|V(G)|}<\frac{\alpha(H)}{|V(H)|}$ and $G$ is disconnected.

Proof. If $E(H)=\emptyset$, the result is obvious, so we assume that $E(H) \neq \emptyset$. By Lemma 2.1 and the MIS-normality of $G^{\prime} \times H$, we have the following inequality

$$
\frac{\alpha(G \times H)}{|V(G)||V(H)|} \leq \frac{\alpha\left(G^{\prime} \times H\right)}{\left|V\left(G^{\prime}\right)\right||V(H)|}=\max \left\{\frac{\alpha(G)}{|V(G)|}, \frac{\alpha(H)}{|V(H)|}\right\} \leq \frac{\alpha(G \times H)}{|V(G)||V(H)|},
$$

yielding

$$
\frac{\alpha(G \times H)}{|V(G)||V(H)|}=\frac{\alpha\left(G^{\prime} \times H\right)}{\left|V\left(G^{\prime}\right)\right||V(H)|}=\max \left\{\frac{\alpha(G)}{|V(G)|}, \frac{\alpha(H)}{|V(H)|}\right\} .
$$

For every $\sigma \in \operatorname{Aut}(G)$, it is clear that $\sigma\left(G^{\prime}\right) \times H$ is MIS-normal. Let $S$ be a maximum independent set of $G \times H$. By Lemma 2.1] and (5), $S \cap\left(\sigma\left(G^{\prime}\right) \times H\right)$ is a maximum independent set of $\sigma\left(G^{\prime}\right) \times H$. Clearly, for each $a \in \partial_{G}(S)$, there is a $\sigma \in \operatorname{Aut}(G)$ such that $a \in \sigma\left(G^{\prime}\right)$. We therefore have that $\left|\partial_{H}(a, S)\right|=|H|$ or $\alpha(H)$ for each $a \in \partial_{G}(S)$. In the following we distinguish three cases to complete the proof.

Case 1: $\left|\partial_{H}(a, S)\right|=|V(H)|$ for every $a \in \partial_{G}(S)$. By (15), we obtain that $\left|\partial_{G}(S)\right|=\alpha(G)$. Since $E(H) \neq \emptyset, \partial_{G}(S)$ is an independent set of $G$. This implies that $S=\partial_{G}(S) \times H$.

Case 2: $\left|\partial_{H}(a, S)\right|=\alpha(H)$ for every $a \in \partial_{G}(S)$. By (15), we have that $\partial_{G}(S)=G$,

$\frac{\alpha(H)}{|V(H)|} \geq \frac{\alpha(G)}{|V(G)|}$ and $\partial_{H}(a, S)$ is a maximum independent set of $H$ for every $a \in G$. Let $a$ be a fixed vertex of $G$, and set

$$
C=\left\{c \in G: \partial_{H}(c, S)=\partial_{H}(a, S)\right\}
$$

If $C=G$, then $S=G \times \partial_{H}(a, S)$. If $C \neq G$, then choose $d \in G-C$ and $c \in C$. Since $\partial_{H}(c, S) \neq \partial_{H}(d, S)$, there exists $u \in \partial_{H}(c, S)$ and $v \in \partial_{H}(d, S)$ such that 
$(u, v) \in E(H)$ and $[(c, u),(d, v)] \notin E(G \times H)$. This implies that $(c, d) \in E(G)$ and thus $\mathrm{G}$ is disconnected.

Case 3: $\left|\partial_{H}(a, S)\right|=|V(H)|$ and $\left|\partial_{H}(b, S)\right|=\alpha(H)$ for some $a, b \in \partial_{G}(S)$. By (5), $\frac{\alpha(H)}{|V(H)|}=\frac{\alpha(G)}{|V(G)|}$ and $\alpha(G \times H)=\alpha(G)|V(H)|=\alpha(H)|V(G)|$. Set

$$
C=\left\{c \in G:\left|\partial_{H}(c, S)\right|=|V(H)|\right\} \text { and } D=\left\{d \in G:\left|\partial_{H}(d, S)\right|=\alpha(H)\right\} .
$$

Since $E(H) \neq \emptyset$, it is clear that $C$ is an independent set of $G$ and $(c, d) \notin E(G)$ for every $c \in C$ and $d \in D$. So $N_{G}[C] \subseteq V(G)-D$. Moreover,

$$
|S|=\alpha(H)|V(G)|=|C||V(H)|+|D| \alpha(H)
$$

Thus $\frac{|C|}{\left|N_{G}[C]\right|} \geq \frac{|C|}{|V(G)|-|D|}=\frac{\alpha(H)}{|V(H)|}=\frac{\alpha(G)}{|V(G)|}$. By Proposition $2.3, \frac{|C|}{\left|N_{G}[C]\right|}=\frac{\alpha(G)}{|V(G)|}$, that is, $G$ is IS-imprimitive.

This completes the proof.

The following Corollary solves Problem 1.2 in a bit more general setting.

Corollary 3.2 Let $G$ be a vertex-transitive, non-bipartite graph. If $G^{2}$ is MISnormal, then $G^{n}$ is also MIS-normal and IS-primitive for all $n \geq 3$.

Proof. We prove by induction on $n$. Since $G^{2}$ is MIS-normal, by Theorem 2.6, $G$ and $G^{2}$ are both IS-primitive. Assume that $G^{d}$ is MIS-normal and IS-primitive for all $d=2, \ldots, n-1$. We now prove that $G^{n}$ is MIS-normal and IS-primitive. Note that $G^{n}=G^{2} \times G^{n-2}$. Let $G^{\prime}$ be some subgraph of $G^{2}$ that is isomorphic to $G$, for instance, the subgraph induced by the set of vertices $\{(u, u): u \in V(G)\}$. It is clear that

$$
\frac{\alpha\left(G^{\prime}\right)}{\left|V\left(G^{\prime}\right)\right|}=\frac{\alpha(G)}{|V(G)|}=\frac{\alpha\left(G^{2}\right)}{\left|V\left(G^{2}\right)\right|}
$$

and $G^{\prime} \times G^{n-2}$ is isomorphic to $G^{n-1}$. Thus by assumption, $G^{\prime} \times G^{n-2}$ is MISnormal. By Theorem 3.1 and Theorem 2.6, it is easy to see that $G^{n}$ is MIS-normal and IS-primitive. This completes the proof.

Acknowledgement The author is greatly indebted to the anonymous referees for giving useful comments and suggestions that have considerably improved the manuscript. He is grateful also for many valuable discussions with Professor J. Wang and Professor C.J. Zhou. 


\section{References}

[1] M.O. Albertson and K.L. Collins, Homomorphisms of 3-chromatic graphs, Discrete Math., 54 (1985) 127-132.

[2] B. Larose and C. Tardif, Projectivity and independent sets in powers of graphs, J. Graph Theory, 40 (2002) 162-171.

[3] C. Tardif, Graph products and the chromatic difference sequence of vertextransitive graphs, Discrete Math., 185 (1998) 193-200.

[4] P.J. Cameron and C.Y. Ku, Intersecting families of permutations, European J. Comb., 24 (2003) 881-890.

[5] V.P. Mario and J. Vera, Independent and coloring properties of direct products of some vertex-transitive graphs, Discrete Math., 306 (2006) 2275-2281.

[6] P. Frankl, An Erdős-Ko-Rado Theorem for direct products, European J. Combin., 17 (1996) 727-730.

[7] P.K. Jha and S. Klavžar, Independence in direct-product graphs, Ars Combin., 50 (1998) 53-60.

[8] R. Ahlswede, H. Aydinian and L.H. Khachatrian, The intersection theorem for direct products, European J. Combin., 19 (1998) 649-661. 